\title{
A self-consistent chemically stratified atmosphere model for the roAp star 10 Aquilae ${ }^{\star}$
}

\author{
N. Nesvacil ${ }^{1,2}$, D. Shulyak ${ }^{3}$, T. A. Ryabchikova ${ }^{4}$, O. Kochukhov ${ }^{5}$, A. Akberov ${ }^{4}$, and W. Weiss ${ }^{2}$ \\ ${ }^{1}$ Department of Radiotherapy, Medical University of Vienna, Waehringer Guertel 18-20, 1090 Vienna, Austria \\ e-mail: nicole.nesvacil@univie.ac.at \\ ${ }^{2}$ Institute for Astronomy (IfA), University of Vienna, Tuerkenschanzstrasse 17, 1180 Vienna, Austria \\ 3 Institute of Astrophysics, Georg-August University, Friedrich-Hund-Platz 1, 37077 Göttingen, Germany \\ ${ }^{4}$ Institute of Astronomy, Russian Academy of Sciences, Pyatnitskaya 48, 109017 Moscow, Russia \\ 5 Department of Physics and Astronomy, Uppsala University, PO Box 516, 75120 Uppsala, Sweden
}

Received 31 August 2012 / Accepted 5 December 2012

\begin{abstract}
Context. Chemically peculiar A-type (Ap) stars are a subgroup of the CP2 stars that exhibit anomalous overabundances of numerous elements, e.g. $\mathrm{Fe}, \mathrm{Cr}, \mathrm{Sr}$, and rare earth elements. The pulsating subgroup of Ap stars, the roAp stars, present ideal laboratories to observe and model pulsational signatures, as well as the interplay of the pulsations with strong magnetic fields and vertical abundance gradients.

Aims. Based on high-resolution spectroscopic observations and observed stellar energy distributions, we construct a self-consistent model atmosphere for the roAp star 10 Aquilae (HD 176232). It accounts for modulations of the temperature-pressure structure caused by vertical abundance gradients. We demonstrate that such an analysis can be used to determine precisely the fundamental atmospheric parameters required for pulsation modelling.

Methods. Average abundances were derived for 56 species. For $\mathrm{Mg}, \mathrm{Si}, \mathrm{Ca}, \mathrm{Cr}, \mathrm{Fe}, \mathrm{Co}, \mathrm{Sr}, \mathrm{Pr}$, and Nd, vertical stratification profiles were empirically derived using the DDAFIT minimisation routine together with the magnetic spectrum synthesis code SYNTHMAG. Model atmospheres were computed with the LLMODELS code, which accounts for the individual abundances and stratification of chemical elements.

Results. For the final model atmosphere, $T_{\text {eff }}=7550 \mathrm{~K}$ and $\log (g)=3.8$ were adopted. While $\mathrm{Mg}, \mathrm{Si}$, Co, and Cr exhibit steep abundance gradients, $\mathrm{Ca}, \mathrm{Fe}$, and $\mathrm{Sr}$ showed much wider abundance gradients between $\log \tau_{5000}=-1.5$ and 0.5 . Elements $\mathrm{Mg}$ and $\mathrm{Co}$ were found to be the least stratified, while $\mathrm{Ca}$ and Sr showed strong depth variations in abundance of up to $\approx 6$ dex.
\end{abstract}

Key words. stars: abundances - stars: atmospheres - stars: chemically peculiar - stars: fundamental parameters stars: magnetic field

\section{Introduction}

10 Aql (HR 7167, HD 176232, HIP 93179) is one of the brightest rapidly oscillating Ap (roAp) stars. These stars exhibit high-overtone, low-degree, non-radial $p$-mode pulsations with periods in the range of 6-24 min (Kurtz \& Martinez 2000; Alentiev et al. 2012). The most distinguished characteristic of roAp pulsations is the dependence of the radial velocity (RV) amplitude and phase on the particular chemical element/ion. After discovery of stratified abundance distribution in the atmospheres of Ap stars, it became possible to connect the observed pulsational characteristics with the line-depth formation in stratified atmospheres (Ryabchikova et al. 2002). However, to get a self-consistent model atmosphere of an Ap star, one needs to take into account anomalous abundances of most elements of the Mendelejev table and the stratified distribution of those elements, which contribute significantly to the continuum and line opacities. Recently, such modelling was performed for a few roAp stars: $\alpha$ Cir (Kochukhov et al. 2009), HD 24712 (Shulyak et al. 2009), and HD 101065 - Przybylski' star (Shulyak et al. 2010). Saio et al. (2010) showed that the use of the self-consistent model atmopshere of HD 24712 allowed better modelling of pulsations and derivation of the theoretical

^ Table 4 and Figs. 10-12 are available in electronic form at http://www . aanda.org distribution of RV amplitudes and phases over the stellar atmosphere, compatible with the observed RV pulsational characteristics.

A first abundance analysis based on high-resolution spectra was performed by Ryabchikova et al. (2000). The mean magnetic field modulus $1.2 \mathrm{kG}$ was determined by comparison with magnetic spectrum synthesis calculations by Kochukhov et al. (2002). Pulsations in 10 Aql were discovered in a search for stars in the northern hemisphere by Heller \& Kramer (1988). Pulsations in spectral lines were detected by Kochukhov et al. (2002). The star exhibits very sharp spectral lines which is typical for very slow rotators. Being one of the brightest roAp stars, $10 \mathrm{Aql}$ was an excellent target for a photometric campaign with the space telescope MOST (Huber et al. 2008). From their detailed analysis, Huber et al. could derive a lower limit for the rotation period $P_{\text {rot }}>1$ month, which was later confirmed by the study of RV pulsations (Sachkov et al. 2008). The authors performed detailed measurements of RV pulsations in the atmosphere of $10 \mathrm{Aql}$ and found a phase jump in the RV pulsations derived from lines of the rare-earth (REE) elements $\mathrm{Nd}$ and $\mathrm{Pr}$. According to theoretical predictions (Khomenko \& Kochukhov 2009; Sousa \& Cunha 2011), the phase jumps may be associated with the so-called nodal regions. To get a proper pulsation model for any roAp star, one needs to derive a depth dependence of the pulsational characteristics. 
The main goal of the present paper is to obtain the chemical and atmospheric structure of $10 \mathrm{Aql}$, along with global parameters such as radius, mass, and luminosity based on highquality spectroscopic, spectrophotometric, and photometric observations. The paper is organized as follows: spectroscopic observations and data reduction are briefly presented in Sect. 2, the methods of the analysis are given in Sect. 3. The resulting abundances, element stratification, and atmospheric structure calculations are presented in Sect. 4. In Sect. 5 we discuss the importance of the detailed abundance and stratification analysis for atmospheric structure modelling.

\section{Observations}

As $10 \mathrm{Aql}$ is a southern hemisphere object, the star was included in a larger survey for high-resolution spectra of sharp-lined Ap stars with the ESO-VLT UVES spectrograph, 68.D-0254(A) (PI Kochukhov). Dichroic standard settings DIC\#2 centered at $346+580 \mathrm{~nm}$ and $437+860 \mathrm{~nm}$ were used to obtain wavelength coverage from 3030 to $10400 \AA$ with only a few minor gaps. The spectral resolution resulting from the chosen slit width of $0.5 \operatorname{arcsec}$ is $R \approx 80000$, with a signal-to-noise ratio of 450-550. Data reduction of UVES observations (e.g. Ryabchikova et al. 2008) was performed with the ESO UVES pipeline (Ballester et al. 2000). Additional continuum rectification was performed using a dedicated IDL programme.

\section{Methods}

\subsection{Abundance analysis}

As a first step in this investigation, a detailed abundance analysis was made for $10 \mathrm{Aql}$ in order to derive input parameters for the first calculation of an individual model atmosphere with the LLMODELS code by Shulyak et al. (2004). This code takes into account individual abundances for all elements and can additionally accept vertical distributions of chemical elements as input. Abundances were derived from equivalent width measurements of 490 lines obtained with the IDL programme ROTATE, a graphical interface for comparison of observed and synthetic spectra, and a modified version of the WIDTH9 code (Kurucz 1993; Tsymbal 1996), which includes additional magnetic line broadening. Abundances for 56 species were calculated. Atomic line data were extracted from the Vienna Atomic Line Database (VALD II, Kupka et al. 1999; Ryabchikova et al. 1999; Piskunov et al. 1995). For this initial abundance analysis, atmospheric parameters from Ryabchikova et al. (2000) were used, i.e. $T_{\text {eff }}=7650 \mathrm{~K}, \log g=4.0$, and a mean magnetic field modulus of $\langle B\rangle=1.2 \mathrm{kG}$. The results were then used to compute an individual model atmosphere. Comparison of observed $\mathrm{H} \alpha$ line profiles with the synthetic profiles based on this new model revealed the necessity to reduce the effective temperature by $100 \mathrm{~K}$ in order to obtain a better fit. Table 1 lists the resulting abundances of chemical elements. These abundances were then used to identify elements that appear to be vertically stratified in the atmosphere of the star. Elements that revealed systematic differences between abundances derived from lines of different ionization stages are expected to be inhomogeneously distributed in the stellar atmosphere.

\subsection{Stratification analysis}

Vertical abundance stratification is considered to be responsible for the impossibility of modelling cores and wings of strong lines with developed Stark wings with the same abundance. It is also seen as responsible for the impossibility of reproducing both lines belonging to different ionization stages of the same element or high- and low-excitation lines with a chemically homogeneous atmosphere (Ryabchikova et al. 2003). All three effects were detected during the initial abundance analysis and nine elements were included in subsequent further stratification analysis.

Starting from the $T_{\text {eff }}=7550 \mathrm{~K}, \log (g)=4.0$ model atmosphere with a peculiar but vertically homogeneous chemical composition, stratification profiles of $\mathrm{Mg}, \mathrm{Si}, \mathrm{Ca}, \mathrm{Cr}, \mathrm{Fe}$, $\mathrm{Co}$, and $\mathrm{Sr}$ were computed with the DDAFIT IDL-based code (Kochukhov 2007). This code iteratively fits a vertical abundance distribution to an unlimited set of observed spectral lines. The stratification profiles are defined by four parameters: upper atmospheric abundance, abundance in deep atmospheric layers, the vertical position of the abundance jump, and the width of the transition region where abundances change between the two values. All four parameters are optimized simultaneously for one element at a time, but a fixed stratification of other elements can also be taken into account.

The element Co was included in the model calculations only after a first consistent stratified model had been derived by Nesvacil (2009). This is because hyperfine structure data, which are necessary to synthesize observed Co line profiles, were not available at the beginning of our study. Stratification of rare earth elements was derived using a semi-empirical fitting procedure based on observed equivalent widths under the assumption of non-local thermal equilibrium (NLTE). Therefore the shape of derived abundance profiles may deviate from a single-step function. The method was described in Mashonkina et al. (2009) and Mashonkina et al. (2005).

\subsubsection{Line selection}

During this first stratification analysis, large numbers of spectral lines were inspected most carefully to ensure that the best suitable lines with the most accurate atomic parameters available were used. For the stratification analysis, strong and weak lines of different ions are used to sample a wide range of atmospheric layers. Lines showing unusually broadened wings and relatively shallow cores, which were suspected to be a result of vertical element stratification, were also selected.

A list of the atomic parameters used for further analysis is given for each element in Table 4. Atomic line parameters were extracted from VALD using the long-format option for magnetic spectrum synthesis. The original sources of atomic data included in VALD are given in the corresponding table caption.

Part of the line sets selected for stratification analysis in this work correspond to the ones presented by Kochukhov et al. (2006) and Ryabchikova et al. (2008). The latter have studied simultaneously the effects of peculiar $\mathrm{Ca}$ isotopic mixtures on line shapes and stratification profiles in a large number of Ap stars. In the present investigation, the same $\mathrm{Ca}$ lines were used for the analysis, with the exception of lines that would be sensitive to the isotopic mixture. Therefore, ignoring this effect does not have a negative influence on the results of the present study.

In order to estimate which atmospheric layers are sampled by the selected set of lines, formation depths of each line were calculated based on the method proposed by Achmad et al. (1991), which was implemented in our spectrum synthesis code. Based on the calculated contribution functions, a range of atmospheric depths that contribute most to the opacities at given wavelength points was derived for each line profile used in our stratification 

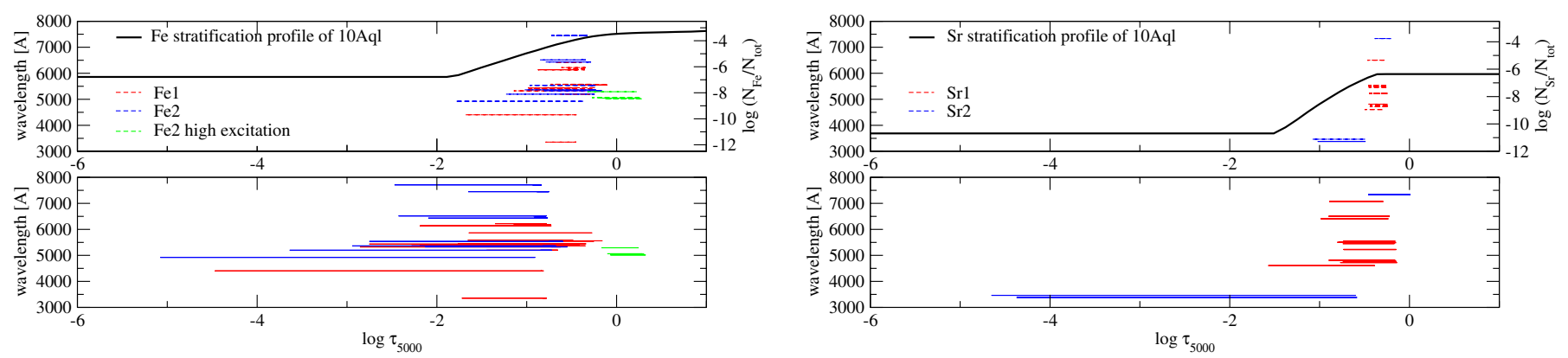

Fig. 1. Range of atmosphere depths that contribute most to the formation of selected line profiles, based on contribution functions according to Achmad et al. (1991) for Fe (left column) and $\mathrm{Sr}$ (right column). Upper panels show example stratification profile and formation depth ranges of selected lines in a stratified model atmosphere. Lower panels show formation depth ranges for lines in a homogeneous model atmosphere.

analysis. Figure 1 shows how these so-called line formation depths change when $\mathrm{Fe}$ or $\mathrm{Sr}$ stratification are introduced into model atmosphere calculations. In the case of Fe, a few lines were available in the high-energy region to sample the upper atmosphere layers and constrain the upper abundance value. High excitation Fe lines are indicated to be useful for sampling lower atmospheric layers. For comparison, a similar plot is shown for a typical $\mathrm{Sr}$ stratification. The available observed $\mathrm{Sr}$ lines appear to be sensitive only to a narrow atmosphere depth region in both the homogeneous and stratified case. Therefore, the derived location of the jump and the deeper atmospheric abundance are considered to be more reliable than the upper atmospheric abundance, which is determined by the depth of the line cores of the strongest lines.

For iron, a large number of observed spectral lines was available. Lines from UV, visual, and near infrared wavelength regions were selected to ensure a better sampling of the atmospheric layers where the abundance jump occurs and to put better constraints on the upper abundance value. In order to test the effect of the choice of the line list on the resulting stratification profile, two Fe distributions were calculated from a complete line list and from a line list excluding UV and infrared lines. The difference between lower atmospheric abundances and the locations and slopes of the abundance jumps was found to be small, i.e. within the formal errors of the fitting routine. The upper atmospheric abundance derived from the line list including strong UV lines was found to be 1.7 dex lower than the one derived from the visual lines only. However, as only two lines in the sample are really sensitive to these layers, the uncertainty of the derived abundance is expected to be of the order of 1 dex.

In order to obtain the best possible sampling of atmosphere layers for all elements, the final stratification line lists aimed to include as many high excitation, UV, and infrared lines as possible, in addition to weak and strong lines from the visual range and lines that exhibit unusually broad wings and shallow cores due to vertical stratification.

\subsubsection{Influence of calculation setup on profile fitting procedure}

For several cases, we find that the fitting routine DDAFIT produces results with large nominal errors for abundances or width of the abundance jump, especially when the atmospheric layers are probed by only a few spectral lines. Therefore, small changes in the model atmosphere setup could have a large effect on the resulting parameters, which might be misinterpreted as being caused by real chemical gradients. A few tests were performed to investigate the behaviour of the code in more detail.

In one experiment, a set of Fe stratification profiles was calculated based on the same model atmosphere but with different depth scales used to compute numerical derivatives in the minimisation part of the code. Here we used optical depth $\log \tau_{5000}$ or column mass $M$. The resulting profiles showed no difference between abundance values in deep atmosphere layers larger than $0.1 \mathrm{dex}$, and location and slope of the transition region were comparable. The upper atmospheric abundance differed by 0.5 dex. This difference could be removed by using a model atmosphere with 288 layers instead of 72 in DDAFIT calculations in $\log \tau_{5000}$. As the increased number of layers also increases overall calculation times by up to a few hours, column mass was used as a depth scale for the rest of the analysis. Finally, results obtained this way are plotted on an optical depth scale. In the case of relatively wide jumps, this transformation results in smoothed shapes of the distribution profile instead of a simple two-step profile, due to the non-linear relation between $\tau_{5000}$ and $M$.

In another experiment, we computed different stratification profiles for seven elements using two model atmospheres with the same temperature, gravity, and chemical composition, but using 80 or 118 layers. The resulting profiles were qualitatively identical for most elements, except for $\mathrm{Ca}, \mathrm{Fe}$, and $\mathrm{Sr}$. In those three cases, the model with higher depth resolution resulted in smaller abundances $(-5,-1.5$, and -2.5 dex $)$ for upper atmospheric layers, while the overall distributions remained the same as for the model sampled on 80 depths. This finding indicates that for these three elements upper abundances are only constrained by a few sensitive lines, and small changes in atmosphere depth resolution might therefore have a large impact on the upper atmospheric abundances derived with DDAFIT. Adjusting the derivative step size used in DDAFIT decreased the formal error of the fitting procedure for the upper atmospheric abundance value below 0.8 dex. The difference of upper atmospheric $\mathrm{Ca}$ and $\mathrm{Fe}$ abundances between the two models thereby decreased as well from -1.4 to -0.7 dex, while it remained $\approx-2.5 \mathrm{dex}$ for Sr. This result indicates that the upper abundance of $\mathrm{Sr}$ is not very well constrained by the available set of spectral lines. Abundance values in higher atmospheric layers are very sensitive to small changes in model atmosphere and calculation setups for all three elements. Other fitting parameters for $\mathrm{Ca}, \mathrm{Fe}$, and $\mathrm{Sr}$ and overall stratification profiles of all other elements do not seem, however, much affected by such changes. During the iterative calculation of self-consistent stratification models, it is sometimes necessary to adjust $T_{\text {eff }}$ to obtain better fits to observed fluxes. Similar to what was described above, another test 
showed that a change of $\pm 100 \mathrm{~K}$ can result in a change of the upper atmospheric abundance by up to a few dex if most available spectral lines are not very sensitive in these layers. Location and slope of the abundance gradient, as well as lower atmospheric abundances were not affected noticeably by such changes in $T_{\text {eff }}$.

\subsubsection{Iterative stratification calculation}

The basic steps of the iterative procedure of atmospheric parameters and abundance determination were outlined in Shulyak et al. (2009). After the first set of stratification profiles had been obtained, new model atmospheres for a set of $\left[T_{\text {eff }}, \log (g)\right]$ pairs were calculated using the derived stratification as input. For testing the quality of various atmosphere models, observed hydrogen line profiles and spectral energy distributions (SEDs) were used. In particular, we made use of spectrophotometric observations by Adelman et al. (1989) and Breger (1976), ultraviolet energy distributions from the International Ultraviolet Explorer (IUE) satellite mission ${ }^{1}$, and spectrophotometric observations obtained by the STIS instrument ${ }^{2}$ mounted at the Hubble Space Telescope. A convergence of the stratification models was reached, when more iterations would not have improved the fit between synthetic line profiles and other observables any further, taking into account the formal error on abundances and step parameters of the DDAFIT procedure.

\section{Results}

\subsection{Abundance analysis}

Mean abundances were computed for a converged stratified model and are summarized in the last three columns of Table 1. For comparison, the results of the abundance analysis based on the initial homogeneous model $\left(T_{\text {eff }}=7550 \mathrm{~K}\right.$ and $\left.\log (g)=4.0\right)$ and an intermediate stratified model with lower temperature are listed (Cols. 2 and 3 respectively). Including stratification in the model atmosphere calculation did not affect the overall abundances of non-stratified elements. The difference between homogeneous and stratified models stayed within 0.1 dex for most elements, which is well within the accuracy of the method of abundance determination by fitting equivalent widths. The comparison of abundances from two stratified models with different temperatures illustrates that the mean abundances of homogeneously distributed elements were not affected by the changes in model structure between different iteration steps of the stratification fitting procedure.

The final converged model contains stratification profiles of nine elements, including REEs. A chemical gradient with increased abundances in upper atmospheric layers was necessary to fit the observed spectral line profiles only for Pr and Nd. All other elements indicate opposite gradients with concentration in deeper layers. A summary of the stratification profiles of all elements in the final model is shown in Fig. 2. Stratification profiles did not change after convergence was reached, compared to the initial homogeneous model.

The largest differences for upper atmospheric abundances, which were found to be the most variable of the four parameters during the iterative process, were observed for $\mathrm{Ca}$ and $\mathrm{Fe}$.

Detailed results of the DDAFIT fitting routine, including formal errors for all four derived stratification parameters (upper or

\footnotetext{
1 http://archive.stsci.edu/iue/

2 http://www.stsci.edu/hst/stis
}

Table 1. Mean abundances and standard deviations derived from observed equivalent widths with the starting model, an intermediate model with $T_{\text {eff }}=7450 \mathrm{~K}$ and $\log (g)=4.0$, and the final model with $T_{\text {eff }}=7550 \mathrm{~K}$ and $\log (g)=3.8$.

\begin{tabular}{|c|c|c|c|c|c|c|c|}
\hline Ion & $\log N / N_{\text {tot }}$ & $\sigma$ & $\log N / N_{\text {tot }}$ & $\sigma$ & $\log N / N_{\text {tot }}$ & $\sigma$ & $\#$ \\
\hline $\mathrm{C}_{\mathrm{I}}$ & -4.21 & 0.06 & -4.28 & 0.06 & -4.24 & 0.06 & 3 \\
\hline OI & -3.89 & 0.38 & -3.96 & 0.40 & -3.92 & 0.38 & 9 \\
\hline $\mathrm{NaI}$ & -6.21 & 0.10 & -6.31 & 0.11 & -6.29 & 0.12 & 2 \\
\hline $\mathrm{Mg}_{\mathrm{I}}$ & -4.60 & 0.12 & * & & $*$ & & 2 \\
\hline $\mathrm{Mg}_{\text {II }}$ & -4.39 & & $*$ & & $*$ & & 1 \\
\hline Al I & -5.84 & 0.03 & -5.93 & 0.03 & -5.90 & 0.03 & 2 \\
\hline Al II & -4.90 & & -4.95 & & -4.91 & & 1 \\
\hline Si I & -4.49 & 0.54 & $*$ & & $*$ & & 7 \\
\hline $\mathrm{S}_{\mathrm{I}}$ & -5.21 & 0.14 & -5.29 & 0.14 & -5.27 & 0.14 & 3 \\
\hline $\mathrm{Ca} I$ & -5.49 & 0.29 & $*$ & & $*$ & & 4 \\
\hline Ca II & -4.67 & 0.03 & $*$ & & $*$ & & 2 \\
\hline Sc II & -9.60 & 0.27 & -9.76 & 0.27 & -9.76 & 0.27 & 6 \\
\hline TiI & -7.26 & 0.34 & -7.37 & 0.35 & -7.37 & 0.35 & 12 \\
\hline Ti II & -7.31 & 0.19 & -7.37 & 0.18 & -7.36 & 0.19 & 19 \\
\hline V II $_{\text {II }}$ & -7.65 & 0.40 & -7.80 & 0.40 & -7.80 & 0.40 & 4 \\
\hline $\mathrm{Cr} \mathrm{I}$ & -5.28 & 0.21 & * & & $*$ & & 33 \\
\hline Cr II & -5.38 & 0.28 & $*$ & & * & & 49 \\
\hline $\mathrm{Mn}$ I & -6.35 & 0.30 & -6.45 & 0.30 & -6.44 & 0.30 & 7 \\
\hline Mn II & -6.42 & 0.02 & -6.51 & 0.08 & -6.50 & 0.09 & 2 \\
\hline $\mathrm{Fe} I$ & -4.45 & 0.34 & $*$ & & $*$ & & 105 \\
\hline $\mathrm{Fe}$ II & -4.09 & 0.36 & $*$ & & $*$ & & 48 \\
\hline CoI & -5.95 & 0.19 & * & & $*$ & & 19 \\
\hline Co II & -5.72 & 0.10 & $*$ & & $*$ & & 2 \\
\hline $\mathrm{Ni}$ I & -6.47 & 0.42 & -6.56 & 0.41 & -6.56 & 0.41 & 13 \\
\hline Ni II & -6.06 & 0.56 & -6.17 & 0.58 & -6.17 & 0.58 & 2 \\
\hline $\mathrm{Cu}$ I & -8.29 & 0.31 & -8.40 & 0.32 & -8.40 & 0.33 & 2 \\
\hline $\mathrm{ZnI}$ & -8.16 & & -8.27 & & -8.27 & & 1 \\
\hline Sr I & -6.94 & 0.19 & $*$ & & $*$ & & 12 \\
\hline Sr II & -7.19 & 0.52 & * & & $*$ & & 2 \\
\hline Y I & -8.48 & 0.08 & -8.59 & 0.08 & -8.58 & 0.08 & 4 \\
\hline Y II & -8.80 & 0.22 & -8.91 & 0.23 & -8.90 & 0.24 & 11 \\
\hline Zr II & -9.38 & 0.17 & -9.51 & 0.18 & -9.51 & 0.18 & 5 \\
\hline Ba II & -9.93 & & -10.05 & & -10.05 & & 1 \\
\hline La II & -10.38 & 0.24 & -10.54 & 0.22 & -10.54 & 0.25 & 3 \\
\hline Ce II & -9.81 & 0.27 & -9.97 & 0.27 & -9.97 & 0.27 & 6 \\
\hline Ce III & -6.75 & 0.34 & -6.88 & 0.37 & -6.84 & 0.37 & 5 \\
\hline Pr II & -9.36 & & $*$ & & $*$ & & 1 \\
\hline Pr III & -9.04 & 0.19 & $*$ & & * & & 4 \\
\hline $\mathrm{Nd}$ II & -10.27 & 0.36 & $*$ & & $*$ & & 9 \\
\hline $\mathrm{Nd}$ III & -7.32 & 0.05 & $*$ & & $*$ & & 2 \\
\hline Sm II & -9.39 & 0.24 & -9.54 & 0.24 & -9.54 & 0.24 & 7 \\
\hline Sm III & -6.92 & 0.13 & -6.94 & 0.15 & -6.91 & 0.16 & 4 \\
\hline Eu II & -9.91 & 0.17 & -10.06 & 0.17 & -10.04 & 0.17 & 4 \\
\hline Gd II & -9.01 & 0.60 & -9.16 & 0.60 & -9.16 & 0.61 & 11 \\
\hline Gd III & -7.65 & & -7.87 & & -7.86 & & 1 \\
\hline Tb III & -8.88 & 0.31 & -9.08 & 0.31 & -9.07 & 0.31 & 7 \\
\hline Dy II & -9.96 & 0.37 & -10.07 & 0.36 & -10.07 & 0.36 & 12 \\
\hline Dy III & -7.45 & 0.26 & -7.63 & 0.26 & -7.62 & 0.26 & 4 \\
\hline Ho II & -10.19 & & -10.26 & & -10.27 & & 1 \\
\hline Ho III & -8.38 & 0.05 & -8.58 & 0.06 & -8.57 & 0.06 & 3 \\
\hline Er II & -9.89 & 0.85 & -10.02 & 0.84 & -10.02 & 0.83 & 2 \\
\hline Er III & -8.10 & & -8.28 & & -8.28 & & 1 \\
\hline Tm II & -10.84 & 0.22 & -10.97 & 0.20 & -10.97 & 0.20 & 2 \\
\hline Tm III & -8.19 & & -8.41 & & -8.40 & & 1 \\
\hline $\mathrm{Yb}$ II & -9.76 & 0.46 & -9.89 & 0.47 & -9.89 & 0.47 & 3 \\
\hline Lu II & -10.38 & 0.14 & -10.53 & 0.14 & -10.52 & 0.14 & 2 \\
\hline
\end{tabular}

Notes. Column "\#” denotes the number of lines measured for each ion. Stratified elements are marked by asterisks.

lower atmospheric abundance, position and width of the abundance jump in the column mass scale used for calculations) are presented in Table 2. 


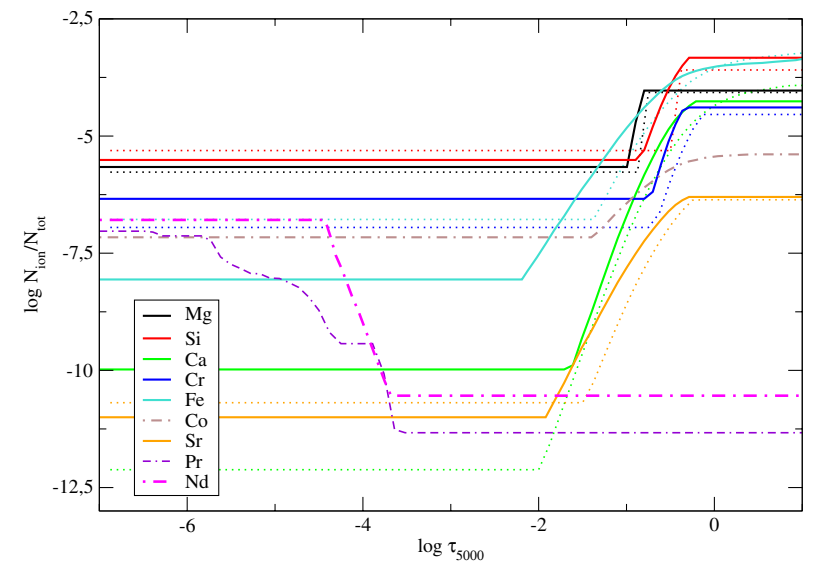

Fig. 2. Stratification profiles of the final model with $T_{\text {eff }}=7550 \mathrm{~K}$ and $\log g=3.8$ (solid lines) and initial homogeneous model (dotted lines). Dash-dotted lines indicate elements that were not included in the initial model but added at a later stage.

Table 2. Stratification parameters for seven elements based on the final model.

\begin{tabular}{cccccc}
\hline \hline & $\begin{array}{c}\log N / N_{\text {up }} \\
\pm\end{array}$ & $\begin{array}{c}\log N / N_{\text {low }} \\
\pm\end{array}$ & $\begin{array}{c}\log M_{\text {step }} \\
\pm\end{array}$ & $\begin{array}{c}\Delta \log M_{\text {step }} \\
\pm\end{array}$ & Dev \\
\hline $\mathrm{Mg}$ & -5.66 & -4.03 & 0.06 & 0.04 & $2.8 \%$ \\
& 0.10 & 0.02 & 0.03 & 0.54 & \\
$\mathrm{Si}$ & -5.51 & -3.33 & 0.17 & 0.16 & $1.7 \%$ \\
& 0.09 & 0.10 & 0.01 & 0.06 & \\
$\mathrm{Ca}$ & -9.98 & -4.26 & -0.02 & 0.56 & $3.2 \%$ \\
& 0.59 & 0.07 & 0.01 & 0.03 & \\
$\mathrm{Cr}$ & -6.30 & -4.42 & 0.21 & 0.02 & $2.4 \%$ \\
& 0.06 & 0.10 & 0.01 & 0.10 & \\
$\mathrm{Fe}$ & -8.06 & -3.16 & -0.14 & 0.95 & $2.6 \%$ \\
& 0.78 & 0.28 & 0.04 & 0.19 & \\
$\mathrm{Co}$ & -7.16 & -5.39 & 0.06 & 0.46 & $3.4 \%$ \\
& 0.16 & 0.41 & 0.05 & 0.36 & \\
$\mathrm{Sr}$ & -11.00 & -6.30 & -0.11 & 0.70 & $2.0 \%$ \\
& 0.73 & 0.22 & 0.04 & 0.17 & \\
\hline
\end{tabular}

Notes. Upper and lower atmospheric abundance values, abundance step position and width in column mass scale, and deviation between observed and synthetic spectra are given. Formal errors of the DDAFIT procedure are given.

Two examples of observed lines, synthetic profiles with the best-fitting homogeneous abundance, and stratified abundances are shown for Fe and Si in Figs. 3, 10-12. Deviation between observed and final synthetic line profiles were between $1.7 \%$ and $3.4 \%$ for all elements.

\subsubsection{Magnesium}

Magnesium stratification profiles did not change significantly between the first and final iterations and were not sensitive to small atmospheric structure changes in any region. Analysis of the contribution functions indicates that the line set is sensitive to a variety of atmospheric depths, as it includes two rather strong $\mathrm{Mg}$ II lines in the UV as well as a number of strong infrared lines, in addition to strong lines in the optical spectral region. The final stratification profile shows an abundance jump of $1.7 \mathrm{dex}$, which is needed to reproduce observed line profiles.

\subsubsection{Silicon}

A variety of suitable Si lines were available for stratification analysis, including five high excitation Si II lines useful for probing deeper atmospheric layers, two strong Si II lines sensitive to upper atmospheric abundances, two infrared Si I lines, and a number of Si I and Si II lines that exhibit broad line wings. Throughout the iterative fitting process, the Si distribution remained stable between different iterations. The abundance jump of 2 dex is located in the same optical depth as for the $\mathrm{Cr}$ profile.

\subsubsection{Calcium}

Calcium shows the largest abundance stratification of all elements investigated in $10 \mathrm{Aql}$, with an abundance change of 5.7 dex between lower and upper atmospheric layers. The range of the transition region of the step profile was found to be $\log \tau_{5000}=[-0.2,-1.7]$. The upper atmospheric abundance was very sensitive to small changes in atmospheric structure occuring during the iterative model calculations. As many strong $\mathrm{Ca}$ lines with anomalously strong wings and shallow line cores were included in the analysis, the lower boundary of the abundance jump was constrained with better accuracy than the upper atmospheric abundance.

\subsubsection{Chromium}

Like $\mathrm{Fe}$ and $\mathrm{Si}$, a variety of spectral lines was available for $\mathrm{Cr}$ analysis, including lines with high excitation energies and strong UV lines. The latter were found to be very sensitive to high atmospheric regions up to $\log \tau=-2$ according to the calculated contribution functions. The derived abundance profile remained very stable over all iterations and shows a small abundance gradient of 2 dex occuring in the same depth region as the $\mathrm{Si}$ abundance step.

\subsubsection{Iron}

Of all the investigated elements, the highest number of suitable spectral lines for stratification calculations was found for Fe. Therefore, the line list is expected to sample a large depth range of the stellar atmosphere. Two weak UV lines, two infrared lines, strong and weak lines of different ionization stages, and high excitation Fe II lines were included, and a large final abundance gradient of 4.6 dex was derived. Inspection of the calculated contribution functions, however, revealed that only two strong lines were sensitive to higher atmospheric layers around $\log \tau=2$, while most other lines were mainly found to be formed between $\log \tau=-0.3$ and -1.5 in a stratified atmosphere. The upper atmospheric abundance was therefore very sensitive to small changes in atmospheric structure between different iteration steps. Nevertheless, the lower atmospheric abundance, position, and slope of the abundance gradient did not change significantly between the first and final iterations.

\subsubsection{Cobalt}

The stratification analysis of Co became possible with the availability of better atomic line data, allowing hyperfine structure to be taken into account for in spectrum synthesis. The corresponding data were taken from Pickering (1996) (Co I) and Bergemann et al. (2010) (Co II). This is especially important as Co stratification is expected to be present (as indicated by the results of the abundance analysis), but not very strong. The final 

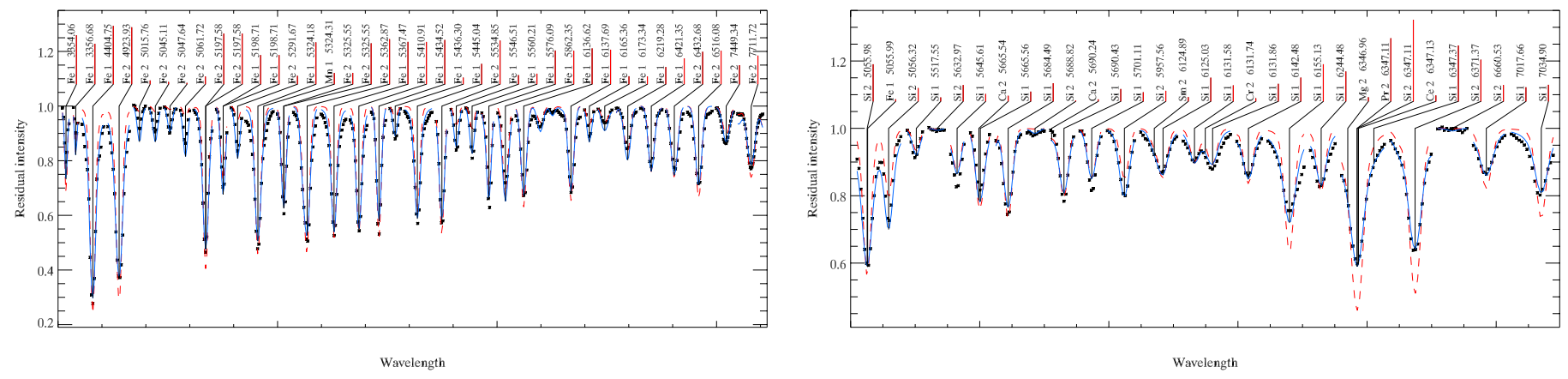

Fig. 3. Fit of observed (black) and synthetic lines with homogeneous (red) and stratified (blue) abundances. Fe is shown in the left column, Si in the right column.

Co stratification was best represented by an abundance jump of 1.8 dex. This small abundance gradient (which was based on a short line list including strong UV lines, as well as intermediate and weak optical lines) proved not to be very sensitive to small changes in model structure.

\subsubsection{Strontium}

Formation depth calculations predicted that the available set of $\mathrm{Sr}$ lines would be sensitive to a very narrow atmospheric region. Throughout the iterative stratification fitting procedure, upper atmospheric abundance values were therefore found to be highly dependent on both small changes in model structure and choice of input parameters for the fitting routine. The final stratification profile of the converged model, however, did not differ significantly from the initial one. A large abundance gradient of 4.7 dex was fit to the observed line profiles by our algorithm. However, as the upper atmospheric abundance is constrained by only a few points in the line profiles of the strongest lines, the real size of the abundance jump might be overestimated.

\subsubsection{Rare earth elements}

For Pr and Nd we performed a NLTE stratification analysis as described in Mashonkina et al. $(2005,2009)$ using a trial-anderror method and the observed equivalent widths of the lines of the first and second ions. As a result, the shape of the abundance profile may differ from a single-step function. Possible magnetic intensification for strong $\mathrm{Nd}$ III lines was approximated by a pseudomicroturbulent velocity of $1.0 \mathrm{~km} \mathrm{~s}^{-1}$. The observed and calculated equivalent widths of $\mathrm{Nd}$ lines are illustrated in Fig. 4.

$\mathrm{Pr}$ and $\mathrm{Nd}$ are the only elements that are enriched in upper atmospheric layers between $\log \tau=-3.6$ and -6.0 and therefore required inclusion of inverted step profiles, compared to the other elements. The observed differences between upper and lower atmospheric abundances were 3.7 dex for $\mathrm{Nd}$ and $4.2 \mathrm{dex}$ for Pr.

\subsection{Fundamental and atmospheric parameters}

Our determination of atmospheric parameters is based on the model fit to the observed energy distributions calibrated to absolute units (see Sect. 3.2.3). We note a discrepancy between space- and ground-based observations, as can be seen from Fig. 5. In particular, data taken from the STIS archive are systematically lower than those of, e.g. broad-band spectrophotometry of Adelman et al. (1989) and Breger (1976) and fluxes computed from Johnson and Geneva photometric systems. In the

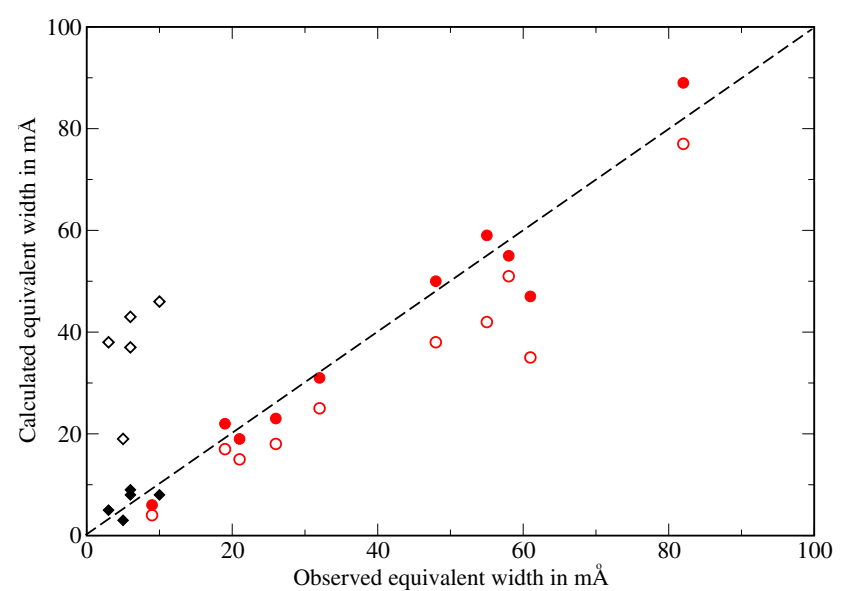

Fig. 4. Comparison between the observed and calculated equivalent widths of Nd II (diamonds) and Nd III (circles) lines. Calculations are made with the derived Nd stratification in LTE (open symbols) and NLTE (filled symbols) approximations.

attempt to fit these datasets, one additional assumption about atmospheric chemistry was made. As follows from the computations of particle diffusion, helium always sinks in subphotosheric layers of A-F stars (Michaud et al. 1979). The same He depletion was found later also in the optically thin layers (Leblanc \& Monin 2004), suggesting that the atmospheres of CP stars of spectral types A-F may well be He-deficient. Having no spectroscopic clues about the true He stratification, we thus considered both He-normal (i.e. solar) and He-weak atmospheres with adopted $\mathrm{He}$ abundance of $\log (\mathrm{He} / \mathrm{H})=-4$ (decreasing He abundance below this value has only a marginal or no effect).

In addition, using observed SEDs and stellar parallaxes allowed us to derive the radius of the star. The latter is obtained by minimising the deviation between observed and predicted fluxes for a given $T_{\text {eff }}$ and $\log (g)$. The parallax $\pi=12.76 \pm 0.29$ mas was taken from van Leeuwen (2007).

Making use of STIS data resulted in $T_{\text {eff }}=7450 \mathrm{~K}, \log (g)=$ 3.8 both for He-normal and He-weak models. Formally, the He-weak model with $\log (g)=3.6$ provided a better fit with slightly lower $\chi^{2}$ compared to the model with $\log (g)=3.8$, but the computed mass of the star is then found to be $0.93 M_{\odot}$, which is too small for an A-type star. Furthermore, the Paschen continuum, which is a good indicator of the atmospheric temperature, is clearly requiring a hotter temperature, which we find to be $T_{\text {eff }}=7550 \mathrm{~K}$. The examples of the model predictions are shown in Fig. 5. The respective fundamental stellar parameters are $R=2.53 \pm 0.06 R_{\odot}, M=1.47 \pm 0.07 M_{\odot}, L=$ $17.78 \pm 0.85 L_{\odot}$ for $T_{\text {eff }}=7450 \mathrm{~K}$ model and $R=2.46 \pm 0.06 R_{\odot}$, 

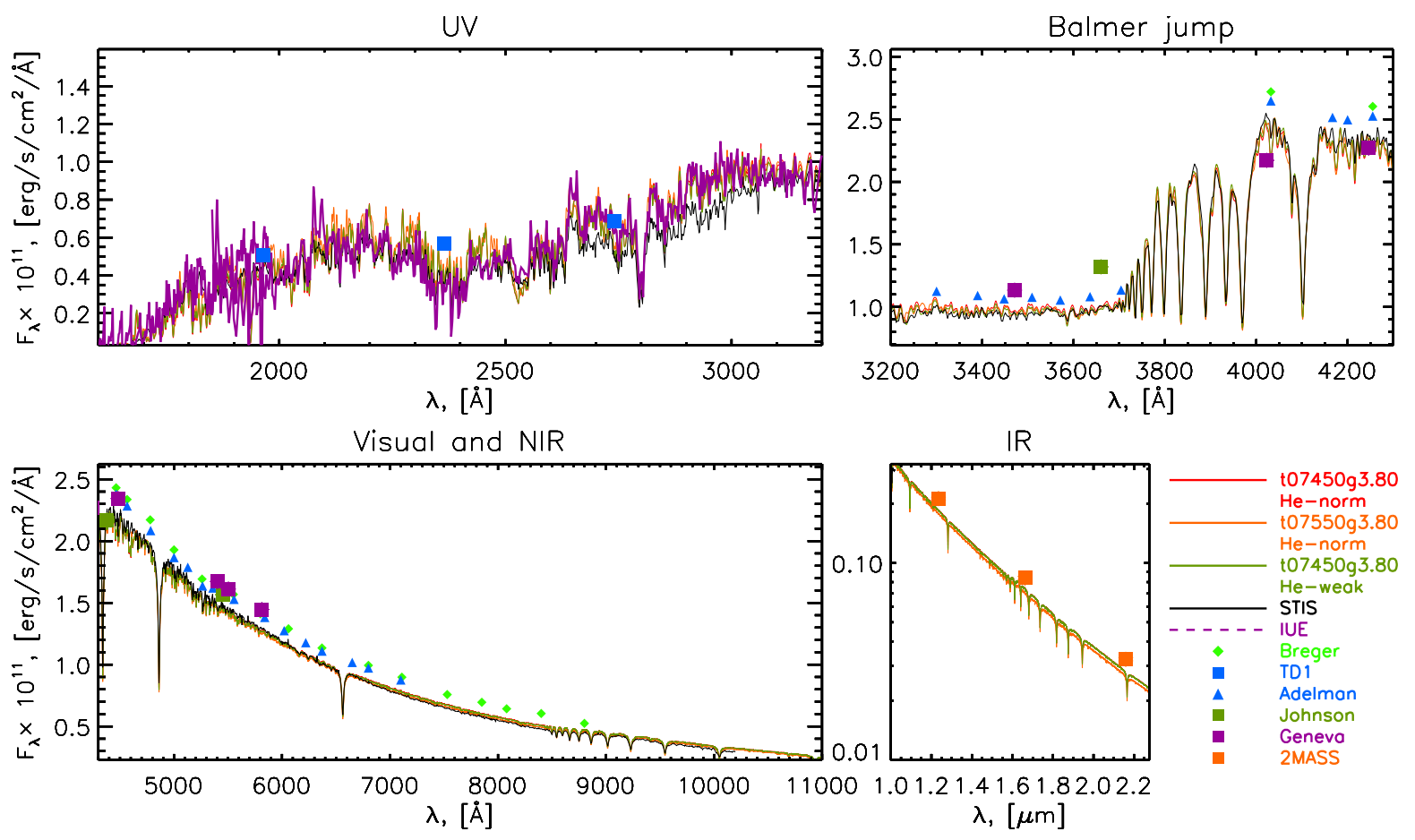

Fig. 5. Comparison between observed and predicted fluxes of $10 \mathrm{Aql}$. The model fit is done to the data obtained with STIS instrument. See online version of this figure for colour coding.

Table 3. Fundamental parameters for He-normal and He-weak models.

\begin{tabular}{lcc}
\hline \hline & \multicolumn{2}{c}{ He-normal } \\
$T_{\text {eff }}$ & $7450 \mathrm{~K}$ & $7550 \mathrm{~K}$ \\
\hline$R$ & $2.67 \pm 0.06 R_{\odot}$ & $2.59 \pm 0.06 R_{\odot}$ \\
$M$ & $1.64 \pm 0.08 M_{\odot}$ & $1.55 \pm 0.07 M_{\odot}$ \\
$L$ & $19.81 \pm 0.90 L_{\odot}$ & $19.66 \pm 0.90 L_{\odot}$ \\
\hline \multicolumn{3}{c}{ He-weak } \\
$T_{\text {eff }}$ & $7400 \mathrm{~K}$ & $7450 \mathrm{~K}$ \\
\hline$R$ & $2.72 \pm 0.06 R_{\odot}$ & $2.67 \pm 0.06 R_{\odot}$ \\
$M$ & $1.70 \pm 0.08 M_{\odot}$ & $1.64 \pm 0.08 M_{\odot}$ \\
$L$ & $20.01 \pm 0.90 L_{\odot}$ & $19.81 \pm 0.90 L_{\odot}$ \\
\hline
\end{tabular}

Notes. $T_{\text {eff }}$ corresponds to the best fits to observed fluxes.

$M=1.39 \pm 0.07 M_{\odot}, L=17.73 \pm 0.88 L_{\odot}$ for the $T_{\text {eff }}=7550 \mathrm{~K}$ model respectively. Note that the error-bars result from parallax accuracy only.

As a next step, we fitted the data obtained from groundbased observations. For this purpose we used spectrophotometric observations by Adelman et al. (1989) and extended them by near infrared points $(\lambda>7000 \AA)$ from Breger (1976). We did so because observations of Adelman cover the region of the Balmer jump and thus provide a homogeneous (in the sense of calibration) set of points in this important spectral region. In this particular case, we find that He-normal models with $T_{\text {eff }}=7450-7550 \mathrm{~K}, \log (g)=3.8$ and He-weak models with $T_{\text {eff }}=7400-7450 \mathrm{~K}, \log (g)=3.8$ provide the best fit. The comparison of the observed and predicted fluxes is shown in Fig. 6. The respective fundamental stellar parameters for the He-normal and He-weak settings are given in Table 3.

Figure 7 illustrates a fit to the observed hydrogen Balmer line profiles for several models. It is seen that a model with $T_{\text {eff }}=7450 \mathrm{~K}$ generally provides an optimal fit to all three Balmer lines. On the other hand, $\mathrm{H} \beta$ and $\mathrm{H} \gamma$ are best fitted with a slightly cooler $T_{\text {eff }}=7400 \mathrm{~K} \mathrm{He}$-weak model.
Taking into account a substantial scatter in the observed energy distributions, the derived atmospheric parameters of the star are $T_{\text {eff }}=7500 \pm 50 \mathrm{~K}, \log (g)=3.8 \pm 0.1$. There are two major spectral regions that play a crucial role in the determination of atmospheric parameters: the Balmer jump (which controls best the value of surface gravity) and the Paschen continuum (which controls best the effective temperature). Therefore, it is important to use observed fluxes from the same data source that cover these two regions. Different calibration schemes applied by different observers could result in a systematic scatter in the derived model parameters. For instance, fluxes obtained by Breger lay slightly above the fluxes by Adelman in the region of the Balmer jump (see, e.g. top right-hand panel of Fig. 5). Using them entirely in model fitting together with IUE data or UV points of Adelman results in a hotter best-fit temperature of $T_{\text {eff }}=7550-7600 \mathrm{~K}$, compared to the one found by fitting data of Adelman extended with NIR points of Breger. Although this is not a large discrepancy, one still should be aware of similar systematics when analysing other stars for which no homogeneous datasets are available.

The difference between space- and ground-based observations results in radius uncertainty of $\Delta R=0.14 R_{\odot}$ (assuming the same $T_{\text {eff }}$ for reference models). This is a rather small discrepancy, which is of the same order as the errors provided by modern interferometry, as found by, e.g. Perraut et al. (2011) for another bright Ap star $\gamma$ Equ. Still, with improving interferometric accuracy, such a scatter between different observed spectrophotometric datasets could become important.

To evaluate the influence of a change in model structure with modified $\mathrm{He}$ abundance on the derived stratification, two sets of profiles were computed using the He-weak and He-normal models with $T_{\text {eff }}=7550 \mathrm{~K}$ and $\log g=3.8$. Results of this comparison are shown in Fig. 8. A small systematic shift of abundance jumps towards lower atmospheric layers is observed when a He-weak composition is assumed. The quality of the fit 

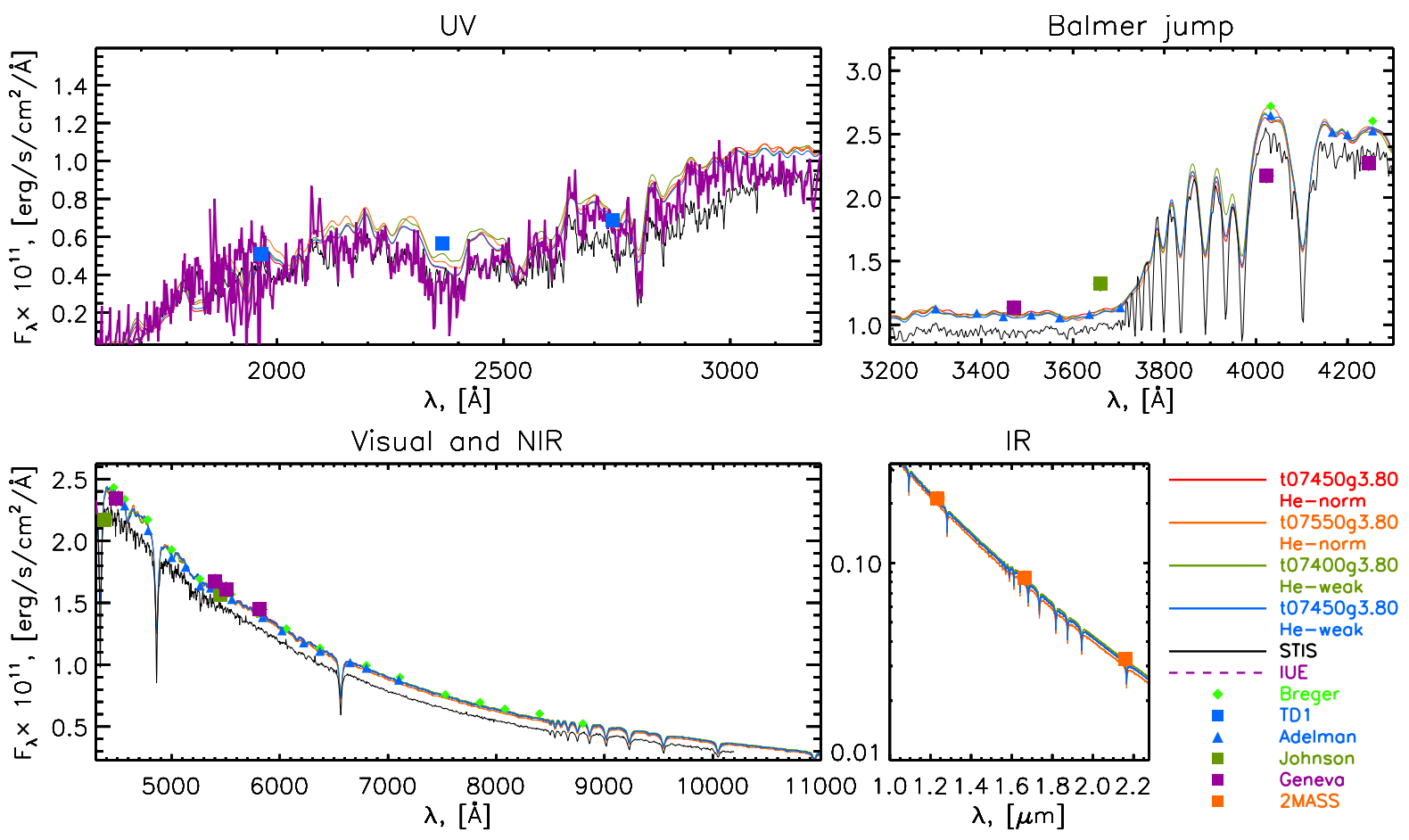

Fig. 6. Same as in Fig. 5, but with the model fit to the ground-based spectrophotometric data of Adelman et al. (1989) and Breger (1976).
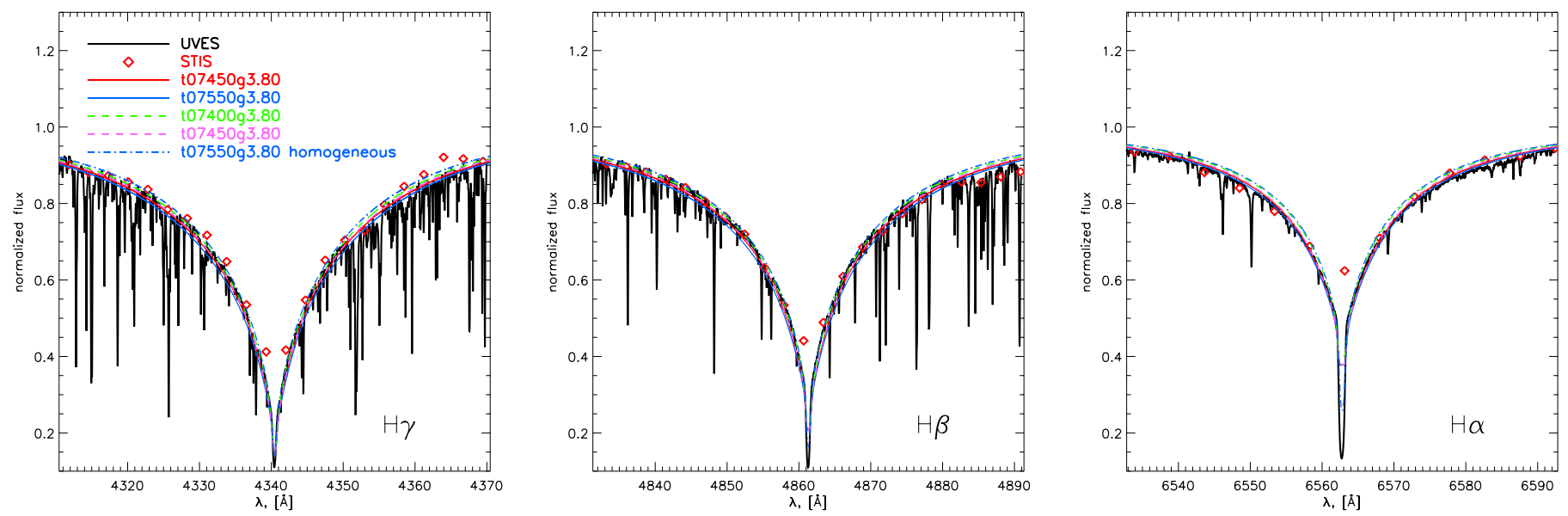

Fig. 7. Observed hydrogen Balmer lines compared to synthetic spectra calculated with different sets of fundamental parameters used during the iterative process. The dashed lines correspond to models computed with depleted He content.

of synthetic line profiles to observations was not found to be different between the two models.

\section{Discussion and conclusions}

We have constructed a model atmosphere for 10 Aql using a selfconsistent iterative approach to derive atmospheric abundances and vertical chemical gradients by fitting models to the observed spectral lines and wide-range energy distributions.

By analysing line profiles in a single spectrum, we were able to derive somewhat mean vertical abundance gradients, i.e. gradients averaged over the visible surface of a star. It is interesting to note that modern Doppler imaging techniques (which rely on the rotationally modulated variability of line profiles) provide us with two-dimensional images of stellar surfaces and reveal nonuniform horizontal distributions of chemical elements and magnetic fields in atmospheres of CP stars. It is therefore natural to think of horizontal inhomogeneities as a result of variable vertical gradients, which depend on the local magnetic field geometry, hydrodynamic flows, and possibly other processes (that we don't yet fully understand). The resulting force that pushes ions upwards or makes them diffuse downwards in the stellar atmosphere becomes variable over the stellar surface. That means that the positions of abundance jumps as well as their amplitudes are then the functions of local surface coordinates, too. Only a self-consistent 3D mapping of stellar atmospheres has the potential of providing vertical and horizontal abundance gradients simultaneously from the same observed data sets. Modern theoretical models of atomic diffusion indeed predict a rather strong sensitivity of element stratification profiles to the geometry of the surface magnetic field (see, for example Leblanc et al. 2009; Alecian \& Stift 2010; Stift \& Alecian 2012).

However, the sharpness of spectral lines and lack of prominent line profile and magnetic field variations imply a very long rotational period for 10 Aql (Ryabchikova et al. 2005; Sachkov et al. 2008). In this situation we are not able to use 


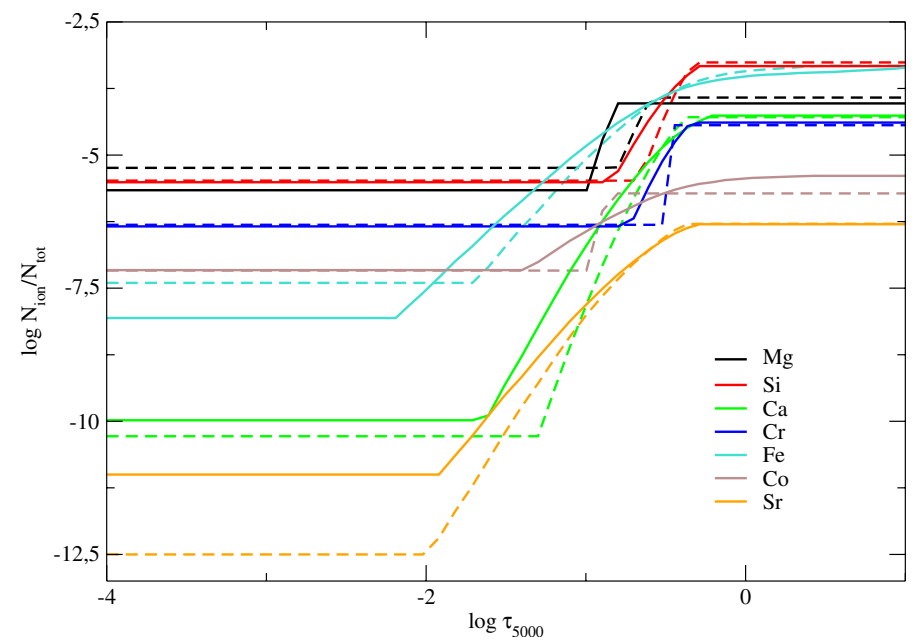

Fig. 8. Comparison of stratification profiles of seven elements computed with He-normal (solid lines) or He-weak (dashed lines) atmosphere models.

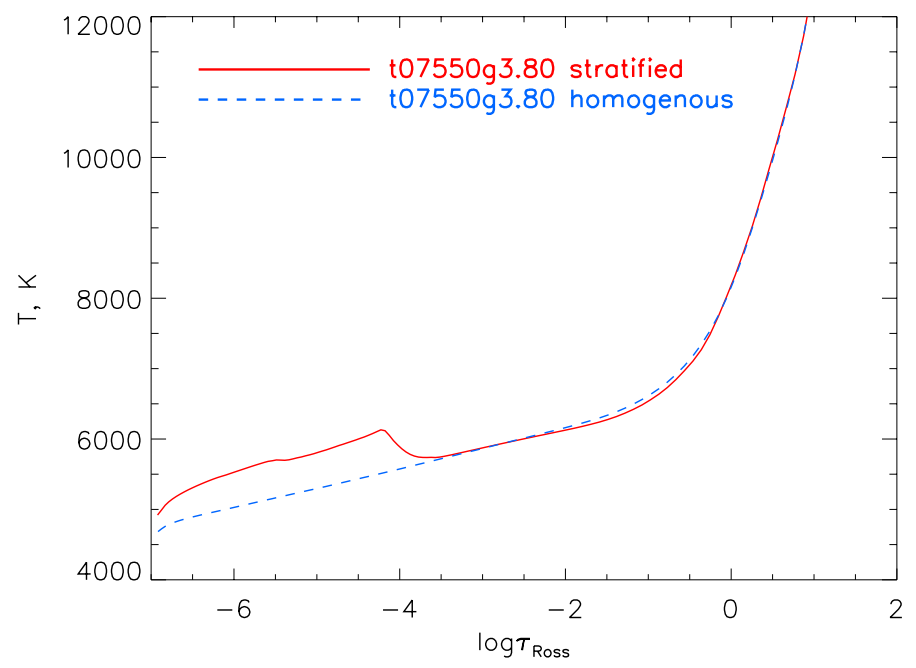

Fig. 9. Temperature structure of models computed with homogeneous and stratified abundance patterns.

Doppler imaging to constrain surface distributions of chemical elements. Very weak long-term line-profile variability detected by (Sachkov et al. 2008) suggests that horizontal abundance gradients in this star are not large or that the star is viewed from nearly the same aspect angle during its rotation cycle. We have estimated that this weak variability would result in a mean abundance variation of $\leq 0.05$ dex. Therefore, we do not expect stratification analysis to be significantly affected by horizontal inhomogeneities.

Our approach of using step-like assumption on derived abundance gradients has obvious limitations. First of all, it is limited to a very narrow atmospheric region, where a given set of atomic lines used in stratification analysis is formed. It naturally cannot predict abundances in optically very thin and thick layers, which we simply do not see with our instruments. Secondly, it is impossible to restore any kind of complex vertical distribution whose shape may strongly deviate from a step-like assumption (see, for example Leblanc et al. 2009; Alecian \& Stift 2010; Stift \& Alecian 2012). However, what is important for us is to see a general trend of element distributions as a function of depths, i.e. whether a given element is being brought upwards or downwards in the line-forming region of a stellar atmosphere and how strong the resulting abundance gradient is. In spite of its limitations, this approach is capable of tracking any kind of systematic as applied to different stars with, e.g. different temperatures and surface magnetic fields.

The detailed analysis of high-resolution spectra ranging from UV to infrared regions indicated that robustness of the inferred vertical stratification profiles is very much dependent on the sensitivity of the selected line set to a wide range of atmospheric depths. As seen, e.g. in our analysis of $\mathrm{Cr}$ stratification, inclusion of high- and low-excitation lines best helps to constrain parameters describing a chemical gradient. However, upper atmospheric abundances are sometimes still only constrained by a few lines that are sensitive to high atmospheric layers. Therefore, the value of the upper atmospheric abundance might vary between an initial homogeneous model atmosphere and a stratified model found after the iterative calculations have converged.

The mean abundances for non-stratified elements changed by about 0.1 dex between the homogeneous starting model and all other stratified models. Abundance variations between different stratified models were negligible. The most striking change between our homogeneous starting model and the final stratified model atmosphere is seen in a systematic shift of the abundance jumps towards higher atmospheric layers in the stratified case.

Between the first and final models, derived stratification profiles were used to calculate synthetic flux distributions to be compared with a large number of observations. This comparison was used for fine tuning of fundamental parameters of 10 Aql. We find that stratification itself has little effect on the synthetic energy distribution of the star compared to models computed with individual and homogeneous abundances. The overall fit and derived radii were almost identical. Therefore, individual abundances appear to be the most important ingredient in the SED modelling, at least in the roAp temperature region. On the other hand, we do find that the observed hydrogen lines could not be reproduced with chemically homogeneous model atmospheres, as illustrated in Fig. 7, where we also show two He-normal models with $T_{\text {eff }}=7550 \mathrm{~K}$, $\log (g)=3.8$ computed with stratified and homogeneous abundances. Therefore, the inclusion of vertical element stratification is preferable when trying to establish an atmosphere model for a single Ap star in order to reproduce spectroscopic and SED observations simultaneously.

Similar to the case of HD 24712 (Shulyak et al. 2009), we find a strong influence of REE opacity in the surface atmospheric structure of stratified models. In particular, strong abundance gradients of $\mathrm{Pr}$ and $\mathrm{Nd}$ shown in Fig. 2 lead to a heating of plasma and appearance of the characteristic temperature jump. This is illustrated in Fig. 9 for He-normal final models. It is interesting to note that such a temperature jump was first empirically predicted by Kochukhov et al. (2002) in order to fit the narrow region of Balmer line profiles between wings and core (the socalled core-wing anomaly). Unfortunately, the temperature jump predicted by our models is located far too high in the atmosphere compared to what was suggested by Kochukhov et al. (2002, see their Fig. 3). We hope that a self-consistent NLTE modelling of REE stratification will help to improve existing models and finally match the predicted position of the temperature jump. Last but not least, the incorporation of an atmospheric model with an inverse temperature gradient from Shulyak et al. (2009) allowed pulsation models of HD 24712 to be improved, as recently computed by Saio et al. (2010). All this suggests that the temperature structure of Ap stars is indeed very much different from the "canonical" $T-\tau$ relation and that the derived temperature jumps are real. 
One of the important results of the present study is the decrease of the $\log (g)$ value from 4.0 to 3.8 once the fitting of SED is introduced in the atmospheric analysis. This is caused by the incorporation of realistic chemistry in model atmosphere computations and the use of homogeneous SED (i.e. STIS data in this particular case), which cover the whole Balmer jump region. A similar decrease of surface gravity was also required in two previous investigations of Ap stars $\alpha$ Cir (Kochukhov et al. 2009) and HD 24712 (Shulyak et al. 2009). In the latter case, recent pulsation modelling by Saio et al. (2010) also suggested that $\log (g)$ of HD 24712 should be lower than what we usually find from photometric calibrations of Ap stars. From our analysis, we conclude that a decrease of $\log (g)$ is consistent with all observations if the appropriate abundance pattern is included in model calculations. On the other hand, decreasing surface gravity does not dramatically change stratification profiles (see our first and final profiles in Fig. 2).

A question that still needs to be answered is the true helium content in the atmospheres of Ap stars. Using available observational material, it is impossible to distinguish between He-normal and He-weak models with high accuracy. Surface temperatures of roAp stars are too low to see He lines. The helium concentration formally influences the quality of the fit to energy distributions, but once plotted against low and moderate resolution data, the difference is difficult to see, except for the Balmer jump region. In addition, hydrogen line profiles are also only marginally affected by He depletion. Pulsation calculations may therefore assume He depletion and still be compatible with all available observations (spectroscopy, spectrophotometry). Improved self-consistent diffusion models are probably the right way to go in the search for a definite answer.

Acknowledgements. Part of this work was supported by the Austrian Fonds zur Förderung wissenschaftlicher Forschung, project P17890 and P22691. D.S. is granted by Deutsche Forschungsgemeinschaft (DFG) Research Grant RE1664/7-1. O.K. is a Royal Swedish Academy of Sciences Research Fellow, supported by the grants from Knut and Alice Wallenberg Foundation and Swedish Research Council. We also acknowledge the use of electronic databases (VALD, SIMBAD, NASA's ADS) and cluster facilities at Vienna Institute for Astronomy and Georg August University Göttingen.

\section{References}

Achmad, L., de Jager, C., \& Nieuwenhuijzen, H. 1991, A\&A, 250, 445 Adelman, S. J., Pyper, D. M., Shore, S. N., White, R. E., \& Warren, W. H., Jr. 1989, A\&AS, 81, 221

Alecian, G., \& Stift, M. J. 2010, A\&A, 516, A53

Alentiev, D., Kochukhov, O., Ryabchikova, T., et al. 2012, MNRAS, 421, L82

Artru, M.-C., Jamar, C., Petrini, D., \& Praderie, F. 1981, AAP, 96, 380

Ballester, P., Modigliani, A., Boitquin, O., et al. 2000, The ESO Messenger, 101, 31

Bergemann, M., Pickering, J. C., \& Gehren, T. 2010, MNRAS, 401, 1334 Bikmaev, I. F., Ryabchikova, T. A., Bruntt, H., et al. 2002, A\&A, 389, 537

Breger, M. 1976, ApJS, 32, 7

Butler, K., Mendoza, C., \& Zeippen, C. J. 1993, J. Phys. B, 26, 4409

Fuhr, J. R., Martin, G. A., \& Wiese, W. L. 1988, J. Phys. Chem. Ref. Data 17, Suppl., 4

Garcia, G., \& Campos, J. 1988, J. Quant. Spectrosc. RA, 39, 477

Den Hartog, E. A., Lawler, J. E., Sneden, C., \& Cowan, J. J. 2003, ApJS, 148, 543

Heller, C. H., \& Kramer, K. S. 1988, PASP, 100, 583

Huber, D., Saio, H., Gruberbauer, M., et al. 2008, A\&A, 483, 239

Ivarsson, S., Litzen, U., \& Wahlgren, M. 2001, Phys. Scr., 64, 455
Jönsson, G., Kröll, S., Persson, A., \& Svanberg, S. 1984, Phys. Rev., A30, 2429 Khomenko, E., \& Kochukhov, O. 2009, ApJ, 704, 1218

Kochukhov, O., Bagnulo, S., \& Barklem, P. S. 2002a, ApJ, 578, L75

Kochukhov, O., Landstreet, J. D., Ryabchikova, T., Weiss, W. W., \& Kupka, F. 2002b, MNRAS, 337, 1

Kochukhov, O., Tsymbal, V., Ryabchikova, T., Makaganyk, V., \& Bagnulo, S. 2006, A\&A, 460, 831

Kochukhov, O., Shulyak, D., \& Ryabchikova, T. 2009, A\&A, 499, 851

Kochukhov, O. P. 2007, Proc. International Conference, held in the Special Astrophysical Observatory of the Russian AS, eds. I. I. Romanyuk, \& D. O. Kudryavtsev, 109

Kupka, F., Piskunov, N. E., Ryabchikova, T. A., Stempels, H. C., \& Weiss, W. W. 1999, A\&AS, 138, 119

Kurtz, D. W., \& Martinez, P. 2000, Balt. Astron., 9, 253

Kurucz, R. L. 1993, SYNTHE Spectrum Synthesis Programs and Line Data (Cambridge: Smithsomian Astrophys. Obs.), CD ROM 18

Kurucz, R. L. 1994, SAO, Cambridge, CD ROM 20-22

Lambert, D. L., \& Luck, R. E. 1978, MNRAS, 183, 79

Leblanc, F., \& Monin, D. 2004, The A-Star Puzzle, 224, 193

Leblanc, F., Monin, D., Hui-Bon-Hoa, A., \& Hauschildt, P. H. 2009, A\&A, 495 937

Martin, G. A., Fuhr, J. R., \& Wiese, W. L. 1988, J. Phys. Chem. Ref. Data, 17, Suppl.3

Mashonkina, L., Ryabchikova, T., \& Ryabtsev, A. 2005, A\&A, 441, 309

Mashonkina, L., Ryabchikova, T., Ryabtsev, A., \& Kildiyarova, R. 2009, A\&A, 495, 297

Michaud, G., Martel, A., Montmerle, T., et al. 1979, ApJ, 234, 206

Nesvacil, N. 2009, Diffusion in atmospheres of magnetic stars, Doctoral Thesis at the University of Vienna

Perraut, K., Brandão, I., Mourard, D., et al. 2011, A\&A, 526, A89

Pickering, J. C. 2006, ApJS, 107, 811

Piskunov, N. E., Kupka, F., Ryabchikova, T. A., Weiss, W. W., \& Jeffery, C. S. 1995, A\&AS, 112, 525

Raassen, A. J. J., \& Uylings, P. H. M. 1998, A\&A, 340, 300

Ralchenko, Yu., Kramida, A. E., Reader, J., \& NIST ASD Team. 2008, NIST Atomic Spectra Database (version 3.1.5), available: http://physics. nist.gov/asd3, NIST, Gaithersburg, MD

Ryabchikova, T. A., Piskunov, N. E., Stempels, H. C., Kupka, F., \& Weiss, W. W. 1999, Phys. Scr. T, 83, 162

Ryabchikova, T. A., Savanov, I. S., Hatzes, A. P., Weiss, W. W., \& Handler, G. 2000, A\&A, 357, 981

Ryabchikova, T., Piskunov, N., Kochukhov, O., et al. 2002, A\&A, 384, 545

Ryabchikova, T., Wade, G. A., \& LeBlanc, F. 2003, Proc. IAU Symp. 210, ASP, 301

Ryabchikova, T., Wade, G. A., Aurière, M., et al. 2005, A\&A, 429, L55

Ryabchikova, T., Ryabtsev, A., Kochukhov, O., \& Bagnulo, S. 2006, A\&A, 456, 329

Ryabchikova, T., Kochukhov, O., \& Bagnulo, S. 2008, A\&A, 480, 811

Sachkov, M., Kochukhov, O., Ryabchikova, T., et al. 2008, MNRAS, 389, 903

Saio, H., Ryabchikova, T., \& Sachkov, M. 2010, MNRAS, 403, 1729

Salih, S., Lawler, J. E., \& Whaling, W. 1985, Phys. Rev., A31, 744

Seaton, M. J., Mihalas, D., \& Pradhan, A. K. 1994, MNRAS, 266, 805

Shulyak, D., Tsymbal, V., Ryabchikova, T., Stütz, Ch., \& Weiss, W. W. 2004, A\&A, 428, 993

Shulyak, D., Ryabchikova, T., Mashonkina, L., \& Kochukhov, O. 2009, A\&A, 499, 879

Shulyak, D., Ryabchikova, T., Kildiyarova, R., \& Kochukhov, O. 2010, A\&A, 520, A88

Smith, G. 1988, J. Phys. B. At. Mol. Opt. Phys., 21, 2827

Smith, G., \& Gallagher, A. 1966, Phys. Rev., 145, 26

Smith, G., \& O'Neil, J. A. 1975, A\&A, 38, 1

Smith, G., \& Raggett D. St. J. 1981, J. Phys., B14, 4015

Sousa, J. C., \& Cunha, M. S. 2011, MNRAS, 414, 2576

Stift, M. J., \& Alecian, G. 2012, MNRAS, 425, 2715

Tsymbal, V. 1996, in M. A. S. S., Model Atmospheres and Spectrum Synthesis, eds. S. J. Adelman, F. Kupka, \& W. W. Weiss, ASP Conf. Ser., 108, 198 van Leeuwen, F. 2007, A\&A, 474, 653

Wiese, W. L., Smith, W. M., \& Miles, B. M. 1969, Atomic Transition Probabilities, NSRDS-NBS 22, Washington, D. C., II

Wilke, R. 2003, Ph.D. Thesis, Heinrich-Heine-Universität, Düsseldorf 
Table 4. Atomic parameters for all lines used in stratification analysis of 10 Aql..

\begin{tabular}{|c|c|c|c|c|c|}
\hline Ion & $\lambda[\AA]$ & $\log g f$ & $E_{\text {low }}$ & $\gamma_{\text {Stark }}$ & Ref. \\
\hline Mg I & 4702.99 & -0.420 & 4.346 & -4.460 & $\mathrm{Bu}$ \\
\hline Mg I & 4730.02 & -2.409 & 4.346 & approx & JK \\
\hline Mg I & 5172.68 & -0.380 & 2.712 & -5.470 & Wi \\
\hline Mg I & 5183.60 & -0.160 & 2.717 & -5.470 & Wi \\
\hline Mg I & 5528.40 & -0.400 & 4.346 & -4.460 & $\mathrm{Bu}$ \\
\hline Mg I & 5711.08 & -1.833 & 4.346 & approx & $\mathrm{Ku}$ \\
\hline $\mathrm{Mg}$ I & 8806.75 & -0.200 & 4.346 & approx & $\odot$ \\
\hline Mg I & 8923.56 & -1.650 & 5.394 & approx & LL \\
\hline Mg II & 3104.71 & -0.030 & 8.864 & -3.970 & $\mathrm{Ku}$ \\
\hline Mg II & 3104.72 & -1.330 & 8.864 & -3.970 & $\mathrm{Ku}$ \\
\hline Mg II & 3104.80 & -0.190 & 8.864 & -3.970 & $\mathrm{Ku}$ \\
\hline Mg II & 4390.51 & -1.700 & 9.999 & -4.070 & Wi \\
\hline Mg II & 4390.57 & -0.530 & 9.999 & -4.070 & Wi \\
\hline Mg II & 4427.99 & -1.200 & 9.996 & -4.400 & Wi \\
\hline Mg II & 4433.98 & -0.900 & 9.999 & -4.400 & Wi \\
\hline Mg II & 4481.12 & 0.740 & 8.864 & -4.700 & $\mathrm{Ku}$ \\
\hline Mg II & 4481.15 & -0.560 & 8.864 & -4.700 & $\mathrm{Ku}$ \\
\hline Mg II & 4481.32 & 0.590 & 8.864 & -4.700 & $\mathrm{Ku}$ \\
\hline Mg II & 7877.05 & 0.390 & 9.996 & -4.540 & Wi \\
\hline Mg II & 8213.98 & -0.950 & 9.999 & -4.770 & $\odot$ \\
\hline Si I & 5517.55 & -2.384 & 5.080 & 8.380 & $\overline{B R}$ \\
\hline Si I & 5645.03 & -2.400 & 5.614 & approx & NL \\
\hline Si I & 5665.56 & -2.040 & 4.920 & 8.290 & LL \\
\hline Si I & 5684.49 & -1.650 & 4.950 & 8.250 & NL \\
\hline Si I & 5690.43 & -1.870 & 4.930 & 8.300 & NL \\
\hline Si I & 5701.10 & -2.050 & 4.930 & 8.310 & NL \\
\hline Si I & 6125.02 & -1.513 & 5.610 & approx & $\mathrm{sH}$ \\
\hline Si I & 6131.57 & -1.705 & 5.610 & approx & $\mathrm{sH}$ \\
\hline Si I & 6131.85 & -1.685 & 5.610 & approx & $\mathrm{sH}$ \\
\hline Si I & 6142.48 & -1.420 & 5.620 & approx & BR \\
\hline Si I & 6155.13 & -0.770 & 5.619 & approx & BR \\
\hline Si I & 6244.47 & -1.363 & 5.610 & approx & $\mathrm{sH}$ \\
\hline Si I & 7017.66 & -1.070 & 5.870 & approx & $\mathrm{sH}$ \\
\hline Si I & 7034.90 & -0.780 & 5.871 & 8.140 & BR \\
\hline Si II & 5055.98 & 0.440 & 10.074 & 9.040 & AJPP \\
\hline Si II & 5056.31 & -0.359 & 10.074 & 9.030 & NL \\
\hline Si II & 5632.96 & -0.820 & 14.186 & approx & Wil \\
\hline Si II & 5688.81 & 0.000 & 14.186 & approx & Wil \\
\hline Si II & 5957.55 & -0.350 & 10.067 & 8.820 & AJPP \\
\hline Si II & 6347.10 & 0.290 & 8.121 & 9.090 & Wil \\
\hline Si II & 6371.37 & -0.080 & 8.121 & 9.080 & NIST \\
\hline Si II & 6660.53 & 0.230 & 14.504 & approx & Wil \\
\hline Ca I & 4226.72 & 0.244 & 0.000 & -6.030 & SG \\
\hline $\mathrm{Ca} \mathrm{I}$ & 5857.45 & 0.240 & 2.933 & -5.420 & $\mathrm{~S}$ \\
\hline Ca I & 5867.56 & -1.570 & 2.933 & -4.705 & S \\
\hline Ca I & 6162.17 & -0.090 & 1.899 & -5.320 & SN \\
\hline Ca I & 6163.75 & -1.286 & 2.521 & -4.998 & SR \\
\hline $\mathrm{Ca} I$ & 6169.04 & -0.797 & 2.523 & -4.997 & SR \\
\hline Ca I & 6169.56 & -0.478 & 2.526 & -4.994 & SR \\
\hline Ca I & 6455.59 & -1.340 & 2.523 & -6.072 & S \\
\hline $\mathrm{Ca} \mathrm{I}$ & 6471.66 & -0.686 & 2.526 & -6.072 & SR \\
\hline Ca II & 5021.13 & -1.207 & 7.515 & -4.612 & TB \\
\hline Ca II & 6456.87 & 0.410 & 8.438 & -3.711 & TB \\
\hline Ca II & 8248.79 & 0.556 & 7.515 & -4.600 & TB \\
\hline
\end{tabular}

Notes. Abbreviations of references correspond to different internal line lists of VALD and individual line lists with observed parameters. When broadening constants were absent in VALD, approximations were used. Stark data for Ca-Co are taken from Kurucz (1994). Stark data for rare earth elements are taken from Hartog et al. (2003). Abbreviations for references are as follows: Bu: Butler et al. (1993); JK: Jönsson et al. (1984); Wi: Wiese et al. (1969); Ku: Kurucz (1993); ๑: experimental comparison with solar spectrum and HD73666, T. Ryabchikova, L. Fossati (priv. comm.); LL: Lambert \& Luck (1978); BR: Bikmaev et al. (2002); NL: Kurucz (1993); sH: solar: Si=7.55 Holweger Model, E. Luck (priv. comm.); AJPP: Artru et al. (1981); Wil: Wilke (2003); NIST: Ralchenko et al. (2008); SG: Smith \& Gallagher (1966); S: Smith (1988); SN: Smith \& O'Neil (1975); TB: Seaton et al. (1994); SR: Smith et al. (1981); GF: Kurucz (1994); MFW: Martin et al. (1988); RU: Raassen \& Uylings (1998); V2: various sources - see details in VALD publications Piskunov et al. (1995); Kupka et al. (1999); Ryabchikova et al. (1999); FMW: Fuhr et al. (1988); SLW: Salih et al. (1985); T83av: average value (Co II) from CUNJ and SLW for the lines with $\log (g f)>-1.0$; Bh: Kurucz (1993); GC: Garcia \& Campos (1988); HLSC: Hartog et al. (2003); RRKB: Ryabchikova et al. (2006); ILW: Ivarsson et al. (2001); ISAN: Ryabtsev (priv. comm.) 
Table 4. continued.

\begin{tabular}{|c|c|c|c|c|c|}
\hline Ion & $\lambda[\AA]$ & $\log g f$ & $E_{\text {low }}$ & $\gamma_{\text {Stark }}$ & Ref. \\
\hline CrI & 3355.73 & -1.107 & 3.847 & -5.118 & GF \\
\hline $\mathrm{Cr} \mathrm{I}$ & 5065.91 & -1.340 & 2.708 & -6.209 & GF \\
\hline Cr I & 5110.74 & -1.289 & 2.709 & -6.210 & $\mathrm{GF}$ \\
\hline $\mathrm{Cr} \mathrm{I}$ & 5204.50 & -0.208 & 0.941 & -6.154 & MFW \\
\hline $\mathrm{Cr} \mathrm{I}$ & 5247.56 & -1.640 & 0.961 & -6.120 & MFW \\
\hline $\mathrm{Cr} \mathrm{I}$ & 5296.69 & -1.400 & 0.983 & -6.120 & MFW \\
\hline $\mathrm{Cr} \mathrm{I}$ & 5345.80 & -0.980 & 1.004 & -6.117 & MFW \\
\hline $\mathrm{CrI}$ & 5368.53 & -1.033 & 3.847 & -6.104 & GF \\
\hline Cr I & 6762.41 & -0.555 & 5.280 & -5.246 & GF \\
\hline $\mathrm{Cr} \mathrm{I}$ & 6762.42 & -0.092 & 5.282 & -5.259 & GF \\
\hline Cr II & 3308.13 & -1.094 & 4.920 & -6.627 & RU \\
\hline Cr II & 3311.93 & -0.510 & 4.156 & -6.648 & RU \\
\hline Cr II & 3338.80 & 0.053 & 6.792 & -5.671 & RU \\
\hline Cr II & 3355.65 & -0.809 & 6.747 & -5.670 & RU \\
\hline Cr II & 3378.33 & -1.045 & 3.104 & -6.716 & RU \\
\hline Cr II & 5024.52 & -1.733 & 6.285 & -6.652 & RU \\
\hline Cr II & 5085.65 & -2.215 & 5.871 & -6.643 & RU \\
\hline Cr II & 5210.82 & -3.259 & 4.042 & -6.720 & RU \\
\hline Cr II & 5210.86 & -2.941 & 3.758 & -6.584 & RU \\
\hline Cr II & 5232.49 & -2.360 & 4.071 & -6.639 & RU \\
\hline Cr II & 5379.79 & -2.081 & 7.331 & -6.558 & RU \\
\hline Cr II & 5678.39 & -1.496 & 6.484 & -6.627 & RU \\
\hline Cr II & 6418.90 & -1.915 & 6.686 & -6.603 & $\mathrm{RU}$ \\
\hline Fe I & 3354.05 & -1.169 & 2.858 & -6.091 & $\mathrm{~V} 2$ \\
\hline $\mathrm{Fe} I$ & 3356.68 & -1.554 & 3.047 & -5.082 & GF \\
\hline $\mathrm{Fe} \mathrm{I}$ & 4404.75 & -0.142 & 1.557 & -6.204 & V2 \\
\hline $\mathrm{Fe} I$ & 5198.71 & -2.135 & 2.223 & -6.185 & V2 \\
\hline $\mathrm{Fe} \mathrm{I}$ & 5324.17 & -0.103 & 3.211 & -5.496 & V2 \\
\hline $\mathrm{Fe} I$ & 5367.46 & 0.443 & 4.415 & -5.133 & V2 \\
\hline $\mathrm{Fe} I$ & 5410.90 & 0.398 & 4.473 & -5.060 & V2 \\
\hline $\mathrm{Fe} I$ & 5434.52 & -2.122 & 1.011 & -6.303 & $\mathrm{~V} 2$ \\
\hline $\mathrm{Fe} \mathrm{I}$ & 5436.29 & -1.540 & 4.386 & -4.997 & V2 \\
\hline $\mathrm{Fe} I$ & 5445.04 & -0.020 & 4.386 & -4.582 & V2 \\
\hline $\mathrm{Fe} I$ & 5546.50 & -1.310 & 4.371 & -6.109 & V2 \\
\hline $\mathrm{Fe} \mathrm{I}$ & 5560.21 & -1.190 & 4.434 & -4.323 & V2 \\
\hline $\mathrm{Fe} \mathrm{I}$ & 5576.08 & -1.000 & 3.430 & -5.491 & $\mathrm{~V} 2$ \\
\hline $\mathrm{Fe} I$ & 5862.35 & -0.058 & 4.549 & -4.582 & GF \\
\hline $\mathrm{Fe} \mathrm{I}$ & 6136.61 & -1.400 & 2.453 & -6.327 & $\mathrm{~V} 2$ \\
\hline $\mathrm{Fe} \mathrm{I}$ & 6137.69 & -1.403 & 2.588 & -6.112 & $\mathrm{~V} 2$ \\
\hline $\mathrm{Fe} \mathbf{I}$ & 6165.36 & -1.474 & 4.143 & -6.156 & V2 \\
\hline $\mathrm{Fe} \mathrm{I}$ & 6173.33 & -2.880 & 2.223 & -6.194 & V2 \\
\hline $\mathrm{Fe} \mathrm{I}$ & 6219.28 & -2.433 & 2.198 & -6.202 & V2 \\
\hline $\mathrm{Fe} \mathrm{I}$ & 6421.35 & -2.027 & 2.279 & -6.310 & V2 \\
\hline Fe II & 4923.92 & -1.504 & 2.891 & -6.583 & RU \\
\hline Fe II & 5015.75 & -0.028 & 10.348 & -5.287 & RU \\
\hline Fe II & 5045.11 & -0.002 & 10.308 & -4.984 & RU \\
\hline Fe II & 5047.64 & -0.235 & 10.308 & -4.976 & RU \\
\hline Fe II & 5061.71 & 0.284 & 10.308 & -5.189 & RU \\
\hline $\mathrm{Fe}$ II & 5197.57 & -2.348 & 3.230 & -6.599 & RU \\
\hline Fe II & 5291.66 & 0.544 & 10.480 & -5.468 & RU \\
\hline Fe II & 5325.55 & -3.324 & 3.221 & -6.603 & RU \\
\hline Fe II & 5362.86 & -2.616 & 3.199 & -6.666 & RU \\
\hline Fe II & 5534.84 & -2.865 & 3.245 & -6.601 & RU \\
\hline Fe II & 6432.68 & -3.687 & 2.891 & -6.687 & RU \\
\hline $\mathrm{Fe}$ II & 6516.08 & -3.432 & 2.891 & -6.686 & RU \\
\hline Fe II & 7449.33 & -3.488 & 3.889 & -6.668 & RU \\
\hline Fe II & 7711.72 & -2.683 & 3.903 & -6.666 & RU \\
\hline CoI & 3502.27 & 0.070 & 0.432 & -6.300 & FMW \\
\hline CoI & 3502.61 & -1.240 & 0.174 & -6.374 & FMW \\
\hline CoI & 4813.46 & 0.050 & 3.216 & -5.627 & GF \\
\hline CoI & 5342.69 & 0.690 & 4.021 & -4.928 & GF \\
\hline CoI & 5347.49 & -0.160 & 4.149 & -4.952 & GF \\
\hline CoI & 6082.42 & -0.520 & 3.514 & -5.474 & GF \\
\hline Co II & 3415.77 & -1.740 & 2.203 & -6.675 & SLW \\
\hline Co II & 3501.71 & -0.970 & 2.203 & -6.673 & T83av \\
\hline
\end{tabular}


N. Nesvacil et al.: A self-consistent stratified atmosphere model for $10 \mathrm{Aql}$

Table 4. continued.

\begin{tabular}{|c|c|c|c|c|c|}
\hline Ion & $\lambda[\AA]$ & $\log g f$ & $E_{\text {low }}$ & $\gamma_{\text {Stark }}$ & Ref. \\
\hline SrI & 4607.32 & -0.570 & 0.000 & approx & $\mathrm{Bh}$ \\
\hline Sr I & 4722.27 & -0.220 & 1.798 & approx & GC \\
\hline Sr I & 4741.91 & -0.410 & 1.775 & approx & GC \\
\hline Sr I & 4784.31 & -0.510 & 1.798 & approx & $\mathrm{Bh}$ \\
\hline SrI & 4811.87 & 0.190 & 1.847 & approx & GC \\
\hline Sr I & 5222.19 & -0.380 & 2.251 & approx & GC \\
\hline Sr I & 5229.26 & -0.330 & 2.259 & approx & $\overline{\mathrm{GC}}$ \\
\hline Sr I & 5450.83 & -0.340 & 2.259 & approx & GC \\
\hline Sr I & 5486.13 & -0.460 & 2.251 & approx & $\mathrm{GC}$ \\
\hline Sr I & 5504.17 & 0.090 & 2.259 & approx & GC \\
\hline Sr I & 5521.76 & -0.060 & 2.251 & approx & GC \\
\hline Sr I & 5540.04 & -0.410 & 2.259 & approx & GC \\
\hline Sr I & 6408.45 & 0.510 & 2.271 & approx & GC \\
\hline SrI & 6503.99 & 0.320 & 2.259 & approx & GC \\
\hline SrI & 7070.07 & -0.030 & 1.847 & approx & GC \\
\hline Sr II & 3380.70 & 0.199 & 2.940 & approx & $\mathrm{Bh}$ \\
\hline Sr II & 3464.45 & 0.487 & 3.040 & approx & $\mathrm{Bh}$ \\
\hline Sr II & 7334.95 & -1.102 & 7.562 & approx & $\mathrm{Bh}$ \\
\hline Nd II & 5130.59 & 0.45 & 1.304 & -5.61 & HLSC \\
\hline Nd II & 5165.13 & -0.74 & 0.680 & approx & HLSC \\
\hline Nd II & 5255.51 & -0.67 & 0.205 & approx & HLSC \\
\hline Nd II & 5293.16 & 0.10 & 0.823 & -5.74 & HLSC \\
\hline Nd II & 5319.82 & -0.14 & 0.550 & -5.82 & HLSC \\
\hline Nd III & 5102.16 & -0.62 & 0.296 & approx & RRKB \\
\hline Nd III & 5294.11 & -0.69 & 0.000 & approx & RRKB \\
\hline Nd III & 5677.18 & -1.45 & 0.631 & approx & RRKB \\
\hline Nd III & 5802.53 & -1.71 & 0.296 & approx & RRKB \\
\hline Nd III & 5845.02 & -1.18 & 0.631 & approx & RRKB \\
\hline Nd III & 5851.54 & -1.55 & 0.460 & approx & RRKB \\
\hline Nd III & 6145.07 & -1.33 & 0.296 & approx & RRKB \\
\hline Nd III & 6327.26 & -1.41 & 0.141 & approx & RRKB \\
\hline Nd III & 6550.23 & -1.49 & 0.000 & approx & RRKB \\
\hline Nd III & 6690.83 & -2.46 & 0.460 & approx & RRKB \\
\hline Pr II & 4222.90 & 0.271 & 0.055 & approx & ILW \\
\hline Pr II & 5322.77 & -0.319 & 0.483 & approx & ILW \\
\hline Pr III & 5299.99 & -0.720 & 0.359 & approx & ISAN \\
\hline Pr III & 6090.01 & -0.871 & 0.359 & approx & ISAN \\
\hline Pr III & 6160.23 & -1.020 & 0.173 & approx & ISAN \\
\hline Pr III & 6195.62 & -1.071 & 0.000 & approx & ISAN \\
\hline Pr III & 7030.39 & -0.929 & 0.359 & approx & ISAN \\
\hline Pr III & 7781.98 & -1.276 & 0.000 & approx & ISAN \\
\hline
\end{tabular}


A\&A 552, A28 (2013)
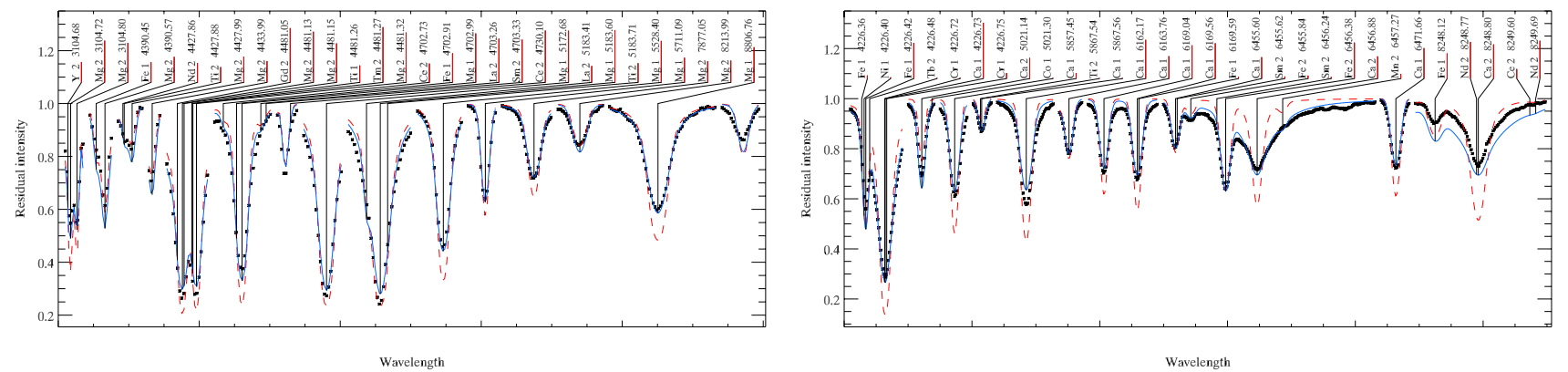

Fig. 10. Fit of observed (black) and synthetic lines with homogeneous (red) and stratified (blue) abundances. Mg is shown in the left column, Ca in the right column.
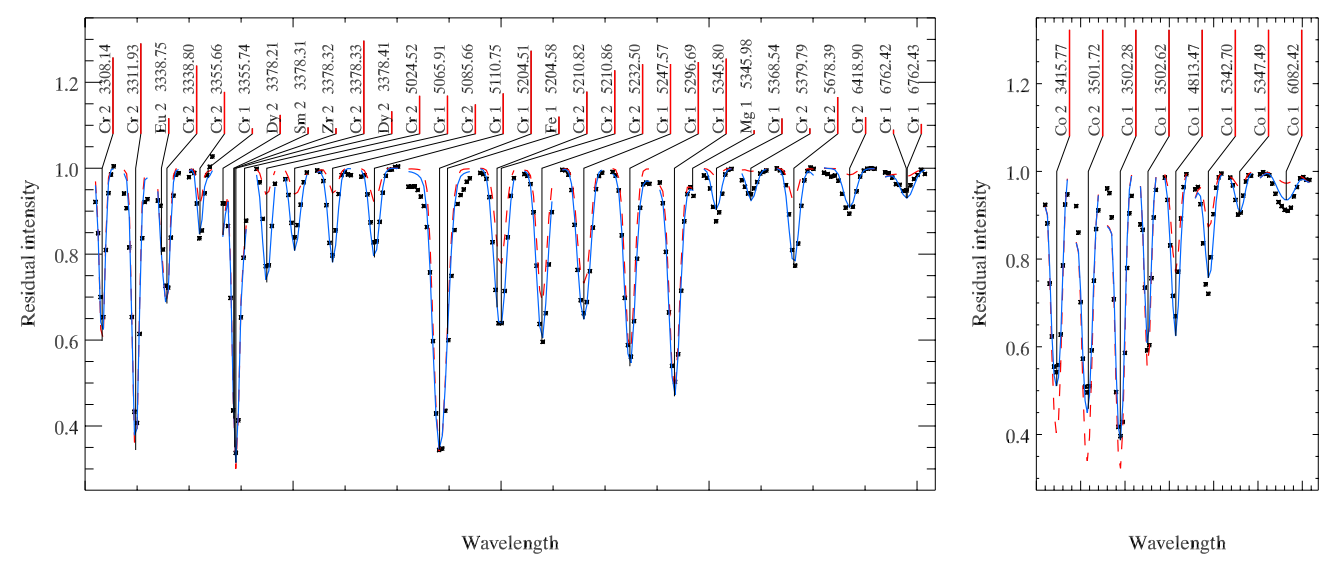

Fig. 11. Fit of observed (black) and synthetic lines with homogeneous (red) and stratified (blue) abundances. Cr is shown in the left column, Co in the right column.

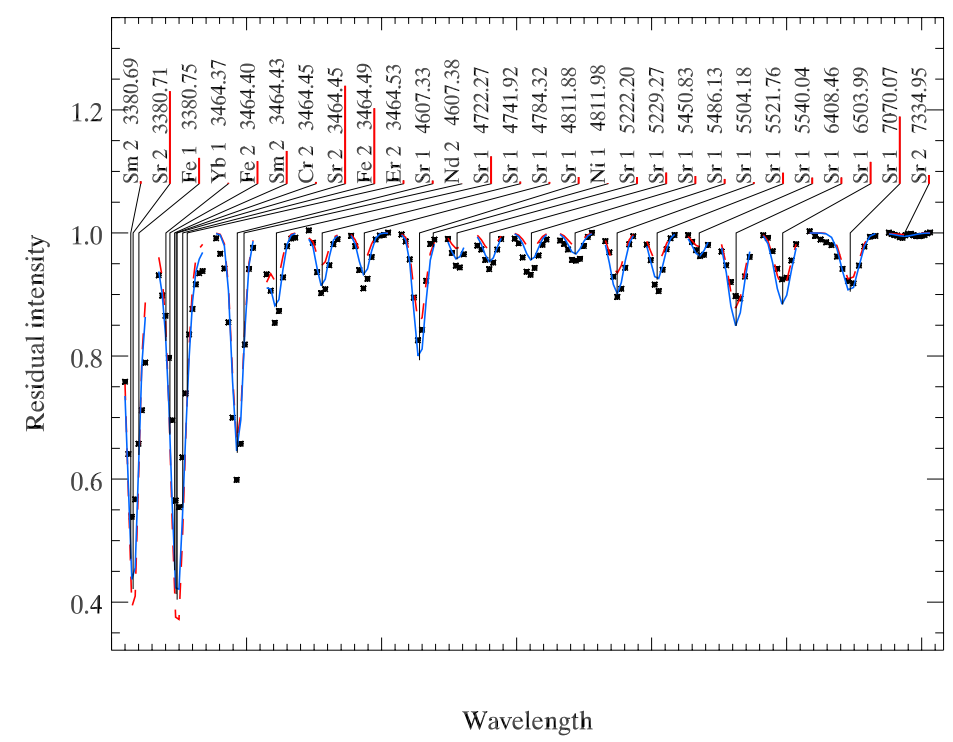

Fig. 12. Fit of observed (black) and synthetic Sr lines with homogeneous (red) and stratified (blue) abundances. 